\title{
EDITORIAL
}

\section{Bisphosphonates for osteoarthritis}

\author{
David A Walsh* and Victoria Chapman \\ See related research by Moreau et al., http://arthritis-research.com/content/13/3/R98
}

\begin{abstract}
Synovitis and subchondral bone turnover are associated with pain in osteoarthritis. Bisphosphonates provide tools for investigating these pathogenic mechanisms and also may have therapeutic potential. Translating preclinical findings into new treatments for human osteoarthritis requires a critical appraisal and refinement of animal models, identification of those pathogenic mechanisms that are amenable to intervention, and pharmacological targeting of those mechanisms in the right people at the right time.
\end{abstract}

Osteoarthritis (OA) is increasingly prevalent as the population ages, and current treatments focus on the relatively short-term relief of symptoms. Clinical trials of disease modification are expensive, requiring prolonged followup of large numbers of participants. In the current global financial climate, there is renewed interest in the OAmodifying potential of existing treatments that have been developed for other indications. Bisphosphonates represent one such class of agent, and the paper by Moreau and colleagues [1] in a recent issue of Arthritis Research $\mathcal{E}$ Therapy provided useful insights into the potential of this class for OA.

Moreau and colleagues reported reduced pain responses when subcutaneous tiludronate was administered to dogs after surgical induction of OA. Furthermore, tiludronate reduced synovitis (effusion, synovial fluid prostaglandin $\mathrm{E}_{2}$ concentration, and lining cell score) and increased subchondral bone thickness. By contrast, an earlier clinical trial of risedronate in patients with OA revealed a disappointing lack of symptom relief compared with placebo [2]. However, lack of efficacy may be explained by the limited potency of risedronate or, in this

*Correspondence: david.walsh@nottingham.ac.uk

Arthritis Research UK Pain Centre, Academic Rheumatology, University of Nottingham Clinical Sciences Building, City Hospital, Hucknall Road, Nottingham NG5 1PB, UK heterogenous disease that varies with time, inadequate targeting to those most likely to benefit. Collectively, these reports raise some important questions about the use of bisphosphonates and general issues for the development of new treatments in OA. How clinically relevant are animal models? Which key pathogenic mechanisms are amenable to intervention? How can treatments be targeted to those mechanisms in the right people at the right time?

Surgical models build on the well-recognized association between $\mathrm{OA}$ and preceding internal derangement [3]. Their predictable onset and rapid development by comparison with spontaneous models facilitate the testing of pharmacological interventions. Most researchers use small-rodent models of OA, although it has been argued that these may inadequately represent the pathogenic processes in humans because of differences in aging and joint biomechanics. Moreau and colleagues partially avoided these problems by studying OA in dogs, although this raises its own ethical issues. The authors maximized the scientific outputs from their study by reporting multiple clinical, biochemical, and histological outcomes. They minimized animal numbers by referring to historical controls rather than using sham surgery. This does, however, hinder interpretation of whether anti-inflammatory effects are directed at OA itself or post-surgical inflammation.

Bisphosphonates could act through several mechanisms. Abnormal bone turnover in OA leads to a zone of osteoporosis beneath the thickened subchondral plate, altered flexibility, and increased microfracture [4]. Osteoclasts mediate the extension of channels from marrow spaces into the non-calcified articular cartilage. The resulting loss of osteochondral integrity exposes subchondral nerves to proinflammatory and algesic factors from the synovial fluid and permits sensory nerve growth into the non-calcified articular cartilage [5]. Furthermore, osteoclasts may reduce $\mathrm{pH}$ at the osteochondral junction, thereby sensitizing and activating sensory nerves through actions on ion channels on their peripheral terminals [6].

The data of Moreau and colleagues are consistent with effects of bisphosphonates on subchondral bone turnover but also suggest possible actions on synovitis. Inflammation is a common feature of OA and is associated both 
with symptoms and with structural progression [7]. Inflammation in OA synovium is characterized by a predominance of macrophages, which can be targeted by bisphosphonates [8]. However, anti-inflammatory cyclooxygenase inhibitors and glucocorticosteroids have only short-term effects on pain in humans. This may be because the catabolic effects of these inhibitors and glucocorticosteroids offset potential long-term clinical benefit, and the possibility that bisphosphonates may protect joint structure while inhibiting inflammation deserves further study.

The quest for structural disease modification in OA has focused largely on joint space narrowing, indicative of cartilage loss and meniscal extrusion, and osteophytosis. However, each of these defines structures that may not be direct sources of pain. The data of Moreau and colleagues support the findings of other groups indicating that structural treatments, despite having little effect on chondropathy or osteophytosis, could reduce pain [9]. Magnetic resonance imaging features more closely associated with pain in OA include synovitis and bone marrow lesions.

Bone marrow lesions identify regions of increased subchondral bone turnover and therefore may provide a biomarker that can predict response to bisphosphonates. Laslett and colleagues [10] recently reported a placebocontrolled trial of the potent parenteral bisphosphonate, zoledronic acid, and showed improved pain in people with $\mathrm{OA}$ and bone marrow lesions. It remains to be determined, however, whether bisphosphonates act exclusively through subchondral bone turnover or whether they may also be effective if targeted to people during OA-associated synovitis.

Animal models reflect the understanding of the pathogenesis of $\mathrm{OA}$ at the time they were developed. Investigating subchondral bone changes and synovitis requires a critical use and refinement of these models. A better understanding of how they reflect the clinical spectrum of OA will facilitate the translation of novel treatments to clinical practice. The bisphosphonate story continues to evolve and there remains optimism that it may lead to targeted treatments with greater effectiveness in the near future.

\section{Abbreviation}

OA, osteoarthritis.

\section{Competing interests}

The authors declare that they have no competing interests.

Published: 30 September 2011

\section{References}

1. Moreau M, Rialland P, Pelletier JP, Martel-Pelletier J, Lajeunesse D, Boileau C, Caron J, Frank D, Lussier B, Del Castillo JR, Beauchamp G, Gauvin D, Bertaim T, Thibaud D, Troncy E: Tiludronate treatment improves structural changes and symptoms of osteoarthritis in the canine anterior cruciate ligament model. Arthritis Res Ther 2011, 13:R98.

2. Bingham CO 3rd, Buckland-Wright JC, Garnero P, Cohen SB, Dougados M, Adami S, Clauw DJ, Spector TD, Pelletier JP, Raynauld JP, Strand V, Simon LS, Meyer JM, Cline GA, Beary JF: Risedronate decreases biochemical markers of cartilage degradation but does not decrease symptoms or slow radiographic progression in patients with medial compartment osteoarthritis of the knee: results of the two-year multinational knee osteoarthritis structural arthritis study. Arthritis Rheum 2006, 54:3494-3507.

3. Poole R, Blake S, Buschmann M, Goldring S, Laverty S, Lockwood S, Matyas J, McDougall J, Pritzker K, Rudolphi K, van den Berg W, Yaksh T: Recommendations for the use of preclinical models in the study and treatment of osteoarthritis. Osteoarthritis Cartilage 2010, 18 (Suppl 3):S10-S16.

4. Buckland-Wright JC, Messent EA, Bingham CO 3rd, Ward RJ, Tonkin C: A 2 yr longitudinal radiographic study examining the effect of a bisphosphonate (risedronate) upon subchondral bone loss in osteoarthritic knee patients. Rheumatology (Oxford) 2007, 46:257-264.

5. Walsh DA, McWilliams DF, Turley MJ, Dixon MR, Franses RE, Mapp PI, Wilson D: Angiogenesis and nerve growth factor at the osteochondral junction in rheumatoid arthritis and osteoarthritis. Rheumatology 2010, 49:1852-1861.

6. Yoneda T, Hata K, Nakanishi M, Nagae M, Nagayama T, Wakabayashi H, Nishisho T, Sakurai T, Hiraga T: Involvement of acidic microenvironment in the pathophysiology of cancer-associated bone pain. Bone 2011, 48:100-105.

7. Sellam J, Berenbaum F: The role of synovitis in pathophysiology and clinical symptoms of osteoarthritis. Nat Rev Rheumatol 2010, 6:625-635.

8. Roelofs AJ, Thompson K, Ebetino FH, Rogers MJ, Coxon FP: Bisphosphonates: molecular mechanisms of action and effects on bone cells, monocytes and macrophages. Curr Pharm Des 2010, 16:2950-2960.

9. Mapp PI, Walsh DA, Bowyer J, Maciewicz RA: Effects of a metalloproteinase inhibitor on osteochondral angiogenesis, chondropathy and pain behavior in a rat model of osteoarthritis. Osteoarthritis Cartilage 2010, 18:593-600.

10. Laslett LL, Dore DA, Quinn SJ, Winzenberg TM, Boon P, Jones G: Zoledronic acid reduces bone marrow lesions and knee pain over one year. Ann Rheum Dis 2011, 70 (Suppl 3):138.

doi:10.1186/ar3448

Cite this article as: Walsh DA, Chapman V: Bisphosphonates for osteoarthritis. Arthritis Research \& Therapy 2011, 13:128. 